\title{
Maneuvering Performance in the Colonial Siphonophore, Nanomia bijuga
}

\author{
Kelly R. Sutherland ${ }^{1, *}$ (D) Brad J. Gemmell ${ }^{2}$, Sean P. Colin ${ }^{3,4}$ and John H. Costello ${ }^{3,5}$ \\ 1 Oregon Institute of Marine Biology, University of Oregon, Eugene, OR 97402, USA \\ 2 Department of Integrative Biology, University of South Florida, Tampa, FL 33620, USA \\ 3 Whitman Center, Marine Biological Laboratory, Woods Hole, MA 02543, USA \\ 4 Marine Biology/Environmental Sciences, Roger Williams University, Bristol, RI 02809, USA \\ 5 Biology Department, Providence College, Providence, RI 02908, USA \\ * Correspondence: ksuth@uoregon.edu
}

Received: 17 June 2019; Accepted: 20 August 2019; Published: 5 September 2019

\begin{abstract}
The colonial cnidarian, Nanomia bijuga, is highly proficient at moving in three-dimensional space through forward swimming, reverse swimming and turning. We used high speed videography, particle tracking, and particle image velocimetry (PIV) with frame rates up to $6400 \mathrm{~s}^{-1}$ to study the kinematics and fluid mechanics of N. bijuga during turning and reversing. N. bijuga achieved turns with high maneuverability (mean length-specific turning radius, $\mathrm{R} / \mathrm{L}=0.15 \pm 0.10$ ) and agility (mean angular velocity, $\omega=104 \pm 41 \mathrm{deg}$. $\mathrm{s}^{-1}$ ). The maximum angular velocity of $N$. bijuga, $215 \mathrm{deg}$. $\mathrm{s}^{-1}$, exceeded that of many vertebrates with more complex body forms and neurocircuitry. Through the combination of rapid nectophore contraction and velum modulation, N. bijuga generated high speed, narrow jets (maximum $=1063 \pm 176 \mathrm{~mm} \mathrm{~s}^{-1} ; 295$ nectophore lengths $\mathrm{s}^{-1}$ ) and thrust vectoring, which enabled high speed reverse swimming (maximum $=134 \pm 28 \mathrm{~mm} \mathrm{~s}^{-1} ; 37$ nectophore lengths s${ }^{-1}$ ) that matched previously reported forward swimming speeds. A 1:1 ratio of forward to reverse swimming speed has not been recorded in other swimming organisms. Taken together, the colonial architecture, simple neurocircuitry, and tightly controlled pulsed jets by N. bijuga allow for a diverse repertoire of movements. Considering the further advantages of scalability and redundancy in colonies, N. bijuga is a model system for informing underwater propulsion and navigation of complex environments.
\end{abstract}

Keywords: turn; reverse; agility; maneuverability; propulsion; Nanomia bijuga

\section{Introduction}

Planktonic marine organisms navigate three-dimensional space to acquire food, avoid predation and reproduce. The colonial siphonophore, Nanomia bijuga, is a cnidarian with multiple swimming units (nectophores) that can swim forward, in reverse, and turn. The presence of multiple jetting units that can be operated individually or simultaneously opens a wider array of swimming maneuvers than is available to organisms that have only one propulsive unit as in medusan jellyfish. Being colonial allows organisms with simple neurocircuitry and morphology to achieve complex movements and is therefore of direct application to designing multi-jet vehicles that are adept at navigating the ocean.

Pulsed jets have been shown to be effective for generating thrust more efficiently than steady jets [1] and have thus become models for underwater vehicles [2] and soft swimming robots [3]. One of the appealing elements of pulsed jets is they allow for maneuvers in small spaces at low speeds more effectively than propellers [4]. Previous biomimetic designs have emulated single-jetters like jellyfish and squid. The presence of multiple jets along the colony axis in N. bijuga can inspire new underwater vehicles that are streamlined (Figure 1a), effective at long-distance cruising, and highly maneuverable due to the strategic placement of jets along the colony axis to produce torque. 


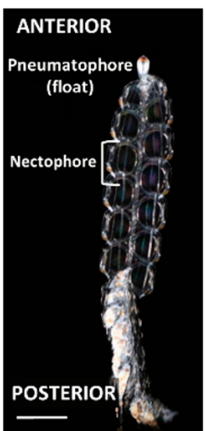

(a)

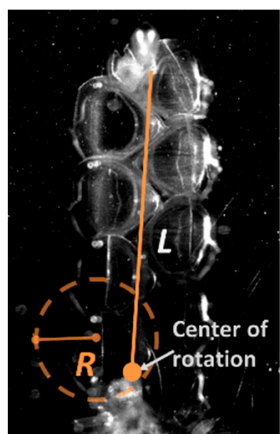

(b)

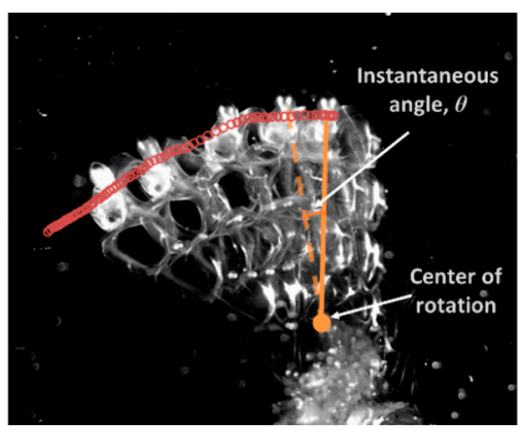

(c)

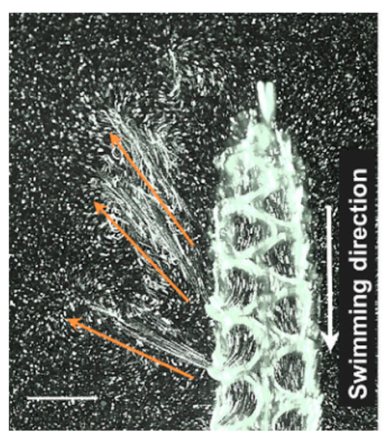

(d)

Figure 1. Maneuverability, agility and reverse swimming in the colonial siphonophore, Nanomia bijuga. (a) Basic anatomy of N. bijuga. Scale bar is $3 \mathrm{~mm}$. (b) Maneuverability (R/L); R, radius of turning path; L, length of nectosome. (c) Agility (angular velocity, $\omega$ ); $\theta$, instantaneous angle. (d) Reverse swimming jets. Orange arrows indicate direction of jets. Scale bar is $5 \mathrm{~mm}$. See Video S1 (turning) and Video S2 (reverse).

N. bijuga first gained the attention of oceanographers because it undertakes long vertical migrations on a diel basis and, owing to the gas-filled pneumatophore, is an important component of the sound scattering layer in much of the worlds' oceans [5]. Detailed studies in the laboratory revealed that in addition to long-distance migrations, N. bijuga is also capable of responding to stimuli (light, mechanical disturbances) by executing rapid maneuvers in three-dimensional space, including turning and reversing [6].

Forward swimming and turning in N. bijuga rely on coordination of multiple swimming units, which are called nectophores. Because there are multiple nectophores in a linear array, the position and orientation of each unit influences its propulsive role [7]. Larger nectophores at the base of the colony are oriented downward and generate most of the straight-swimming thrust. Newer nectophores that have been recently budded at the apex of the colony have a long lever arm and a high angle relative to the colony axis; these nectophores generate torque for turning and very little thrust. At the level of individual nectophores, the integration of motion by the nectophore and velum-a funnel-shaped band of tissue at the jet orifice- direct the fluid to control jetting and refill and allow for high-speed $\left(\sim 1 \mathrm{~m} \mathrm{~s}^{-1}\right)$, narrow jets (1-2 $\left.\mathrm{mm}\right)$ during forward swimming [8].

Maneuvering in three dimensions is achieved through a combination of placement and coordination of the nectophores and changes in velar orientation. At the nectophore-level, the kinematics of jetting and refill are identical during forward swimming and turning. However, during forward swimming, all or most of the nectophores are recruited to swim either asynchronously (steady-state swimming) or synchronously (escape swimming) whereas during turning, typically only one apical (anterior) nectophore fires, leveraging torque due to its position and orientation [7]. Reverse swimming is achieved by the highly maneuverable velum, which directs the fluid in the anterior direction during reversals ([6]; Figure 1d, Video S2). As in forward swimming, all or most of the nectophores are recruited during reverse swimming. Because reverse swimming is executed as an escape maneuver, all nectophores fire synchronously.

Previous studies have quantified forward swimming performance in N. bijuga [7] but turning and reverse swimming have only been described qualitatively [6]. In the present study, our goal was to: (1) quantify forward and reverse swimming performance and (2) to investigate how individual nectophore kinematics, especially in the velum, control fluid motion and contribute to turning and reverse swimming in N. bijuga. Currently, machines cannot match the swimming maneuvers of animals. Quantification of maneuvering performance variables allows for comparisons with other organisms and creates a baseline for improving agility and maneuverability in underwater vehicles. N. bijuga is a model for achieving complex maneuvers with simple, distributed morphology and primitive neuronal control. Though studying fast-swimming organisms with narrow (1-2 mm) high speed jets 
$\left(1 \mathrm{~m} \mathrm{~s}^{-1} ;[8]\right)$ is not without challenges, their transparent bodies provide a unique opportunity for fluid imaging.

\section{Materials and Methods}

N. bijuga colonies were collected in individual containers from docks at Friday Harbor Laboratories, WA, USA, in June 2014, 2016 and 2017 and maintained in running seawater tables at field temperatures $\left(10-12{ }^{\circ} \mathrm{C}\right)$. Over the course of the study over 100 colonies were collected. We obtained 21 turning or reverse swimming sequences from 16 colonies that were in focus and behaving normally. Colonies used in analyses had a mean nectosome length of $13.9 \pm 4.0 \mathrm{~mm}$ (mean $\pm \mathrm{SD}$ ) and $9 \pm 2.7$ nectophores. Nectophores were $3.6 \pm 0.46 \mathrm{~mm}$ in width. Kinematic and fluid mechanic measurements were made with high-speed videography in custom glass vessels within $24 \mathrm{~h}$ of collection [8].

\subsection{Fluid Mechanics}

To examine the relationship between velum kinematics and fluid mechanics of the jet, we used a combination of laser sheet PIV [9] and particle tracking [7]. Images were collected with high speed monochrome video cameras at 500-6400 frames per s (models from Photron Fastcam, Phantom and Edgertronic). For the PIV set-up, $10 \mu \mathrm{m}$ hollow glass beads were seeded into the tank and illuminated with a $<1 \mathrm{~mm}$ thick continuous laser light sheet $(532 \mathrm{~nm}$ ). Image sequences during jetting and refill were selected where the velar aperture was bisected by the laser sheet. Image pairs were subsequently analyzed to examine refill using a cross-correlation PIV algorithm with a multi-pass interrogation window size of $64 \times 64$ pixels down to $32 \times 32$ pixels and $50 \%$ overlap (DaVis 8.3 ) to produce instantaneous velocity vectors and vorticity contours. Instantaneous jet velocities were too high to capture even at shutter speeds of $1 / 10,000 \mathrm{~s}$. Therefore, jet velocities were measured by tracking individual particles from image stacks or measuring particle streaks (ImageJ, NIH, Bethesda, MD, USA) [7].

\subsection{Kinematics}

Whole-colony swimming speeds and kinematics were measured from PIV images where the colony remained in-plane over 1-3 pulse cycles. For turning sequences, this method ensured that motion was in two dimensions to extract accurate angular velocities. For kinematic measurements of individual nectophores, $N$. bijuga colonies were illuminated using a brightfield set-up with a 10× LWD objective [10]. Image stacks were imported into Image to measure morphometric variables, swimming speed, time spent jetting and refilling during each pulse cycle and turning parameters (see below).

\subsection{Turning Performance}

Radius of curvature, $\mathrm{R}$-also defined as maneuverability-was calculated by tracking the $\mathrm{x}, \mathrm{y}$ position of the center of rotation during a turn using ImageJ (Figure 1b; Video S1). The $\mathrm{x}, \mathrm{y}$ positions were then fit with a circle of radius $\mathrm{R}$ based on a least squares approach using the MATLAB routine Curv.m. Length specific turning radius, $\mathrm{R} / \mathrm{L}$, is the radius of curvature divided by the nectosome length.

Instantaneous angular velocity through a turn, $\omega$, also defined as the agility, was calculated by measuring the change in the colony angle, $\theta$, between successive time steps. Colony angle, $\theta$, was defined based on the $\mathrm{x}, \mathrm{y}$ position of colony tip (just below pneumatophore) at two successive time steps and the center of rotation at the base of the nectosome (Figure 1b). Angles were calculated using a custom MATLAB routine based on the dot product and length of the two vectors that defined the angle. The angular velocity, $\omega$, was calculated as the change in instantaneous angle between time steps. Angular acceleration was calculated as the change in instantaneous velocity between time steps. 


\section{Results}

High-speed microvideography with kinematic and fluid mechanical analyses with 21 sequences from $16 \mathrm{~N}$. bijuga colonies showed how turns and reversals were executed.

\subsection{Turning}

Turns in N. bijuga colonies were accomplished by pulsing of a single apical nectophore. Nectophore kinematics and resultant fluid mechanics during turning matched those of straight swimming and are described elsewhere [8]. By pulsing one apical nectophore, colonies were capable of rapid turns with a mean angular velocity, $\omega$, of $104 \pm 41 \mathrm{deg}$. $\mathrm{s}^{-1}$ and a maximum of $215 \pm 90 \mathrm{deg}$. $\mathrm{s}^{-1}$ (Table 1 ). The maximum measured angular velocity for all colonies was $363 \mathrm{deg}$. $\mathrm{s}^{-1}$. The mean peak acceleration was $9795 \pm 7469 \mathrm{deg} \mathrm{s}^{-2}$. Turns were characterized by very little forward motion and a tight turning radius: the average length specific radius of curvature, $\mathrm{L} / \mathrm{R}$, was $0.15 \pm 0.10$ (Table 1). As angular velocity increased, R/L also increased (Figure 2), which was indicative of a trade-off between these two performance variables.

Table 1. N. bijuga turning performance. $\omega$, angular velocity; Acc, acceleration; $R$, radius of curvature; $R / L$, length specific turning radius. Mean values \pm st. dev.; max. and min. values are based on the highest and lowest mean values from all sequences; N, number of colonies; \# seq., number of sequences.

\begin{tabular}{cccccc}
\hline & $\boldsymbol{\omega}\left(\mathbf{d e g ~ s}^{-\mathbf{1}}\right)$ & $\boldsymbol{\omega}_{\max }\left(\mathbf{d e g ~ s}^{\mathbf{- 1}}\right)$ & $\mathbf{A c c}_{\max }\left(\mathbf{d e g ~ \mathbf { ~ s } ^ { - \mathbf { 2 } } )}\right.$ & $\mathbf{R}(\mathbf{m m})$ & $\mathbf{R} / \mathbf{L}$ \\
\hline Mean & $104 \pm 41$ & $215 \pm 90$ & $9795 \pm 7469$ & $1.64 \pm 0.77$ & $0.15 \pm 0.10$ \\
Max & 165 & 363 & 21635 & 3.10 & 0.36 \\
Min & 56 & 122 & 3510 & 0.72 & 0.05 \\
N & 6 & 6 & 6 & 7 & 9 \\
\# seq. & 8 & 8 & 8 & 11 & 11 \\
\hline
\end{tabular}

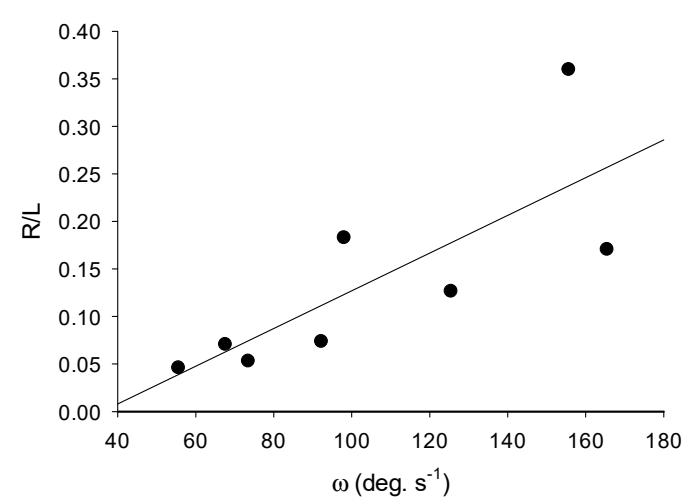

Figure 2. Mean angular velocity, $\omega$, versus length specific radius of turn, $\mathrm{R} / \mathrm{L} ; y=0.002 x-0.0715$; $\mathrm{R}^{2}=0.6 ; p=0.024 ; n=8$.

\subsection{Reverse Swimming}

Reverse swimming was evoked by stimulating the colony tip, near the pneumatophore, and typically was constrained to a single jet cycle but during more vigorous responses, multiple pulses could be evoked (Figure 3a). During reverse swimming, jet velocities reached a maximum of $\sim 1 \mathrm{~m} \mathrm{~s}^{-1}$, equivalent to 295 nectophore lengths $\mathrm{s}^{-1}$, and body speeds reached $134 \mathrm{~mm} \mathrm{~s}^{-1}$ (Table 2), equivalent to 37 nectophore lengths $\mathrm{s}^{-1}$. Reynolds number based on nectophore diameter and swimming speed was $\sim 100$. 


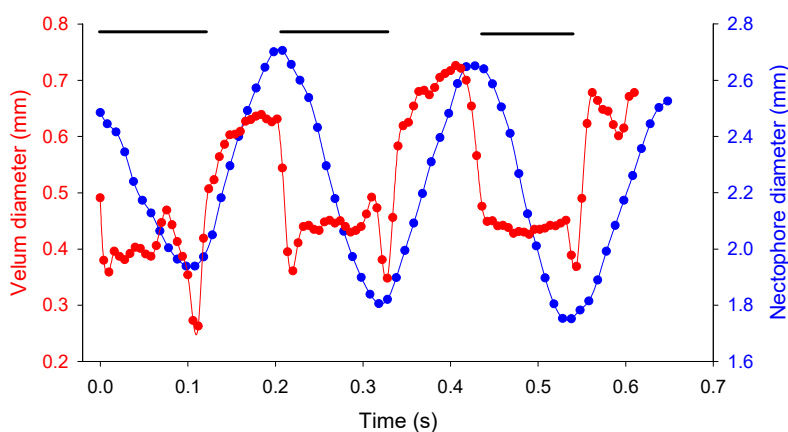

(a)

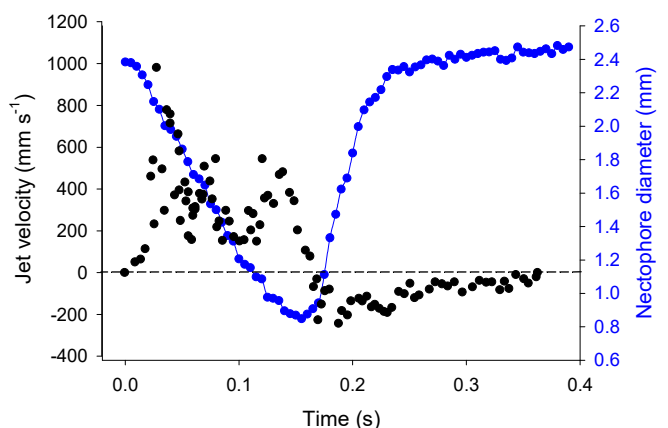

(b)

Figure 3. Orchestration of velum and nectophore kinematics and resultant jet velocities during reverse swimming (a) Time-varying nectophore diameter and velum diameter over three pulse cycles. Black bars indicate jetting period; (b) Time-varying nectophore diameter and jet velocities from particle tracks over one pulse cycle. Jet velocities are negative during refill.

Table 2. N. bijuga reverse swimming performance. N, number of colonies; \# seq., number of sequences.

\begin{tabular}{ccccccc}
\hline & Jetting (s) & Refilling (s) & Total (s) & Ratio & $\begin{array}{c}\text { Max. Jet Speed } \\
\left(\mathbf{m m ~ s}^{-1}\right)\end{array}$ & $\begin{array}{c}\text { Max. Body Speed } \\
\left(\mathbf{m m ~ s}^{-1}\right)\end{array}$ \\
\hline Mean & $0.14 \pm 0.02$ & $0.23 \pm 0.08$ & $0.37 \pm 0.10$ & 0.67 & $1063 \pm 176$ & $134 \pm 28$ \\
Max & 0.10 & 0.10 & 0.21 & 0.43 & 879 & 98 \\
Min & 0.16 & 0.30 & 0.44 & 0.99 & 1260 & 166 \\
N & 6 & 5 & 5 & 5 & 5 & 4 \\
\# seq. & 6 & 5 & 5 & 5 & 5 & 5 \\
\hline
\end{tabular}

During refill, the velum increased in diameter more quickly than the nectophore and fluid velocities slowed owing to the larger velar opening (Figure 3; Video S3). Fluid was pulled in posterior to the nectophore and after entering through the velum, the fluid circulated through the nectophore at a relatively low velocity of $\sim 100 \mathrm{~mm} \mathrm{~s}^{-1}$ (Figures 3 and 4), generating vorticity levels of $\sim 250 \mathrm{~s}^{-1}$ (Figure 4).

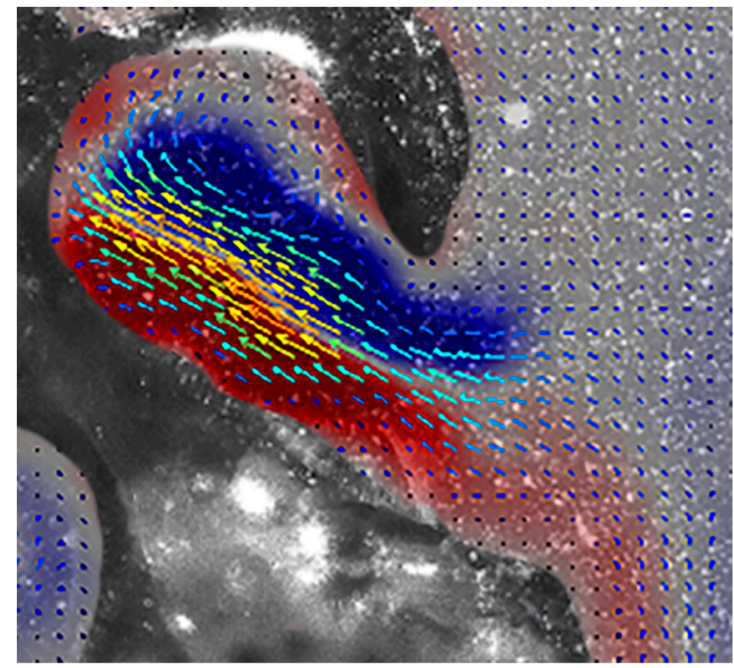

Figure 4. Velocity and vorticity during reverse refill. Fluid is pulled in posterior to the nectophore, potentially enhancing thrust. See Video S3.

Reversals were accomplished through thrust vectoring of the velum (Figure 5). During forward swimming, the velum was oriented posteriorly at an oblique angle to the colony axis. During reverse swimming, the velum reoriented to direct the jet anteriorly. The typical pattern was for the velum to be 
oriented either posteriorly (forward swimming) or anteriorly (reverse swimming) for the duration of the jet but in one instance, we observed the velum reorienting mid-cycle (Figure 5, Video S4). Switching direction mid-pulse is atypical but helps to illustrate the full range of velar orientation.
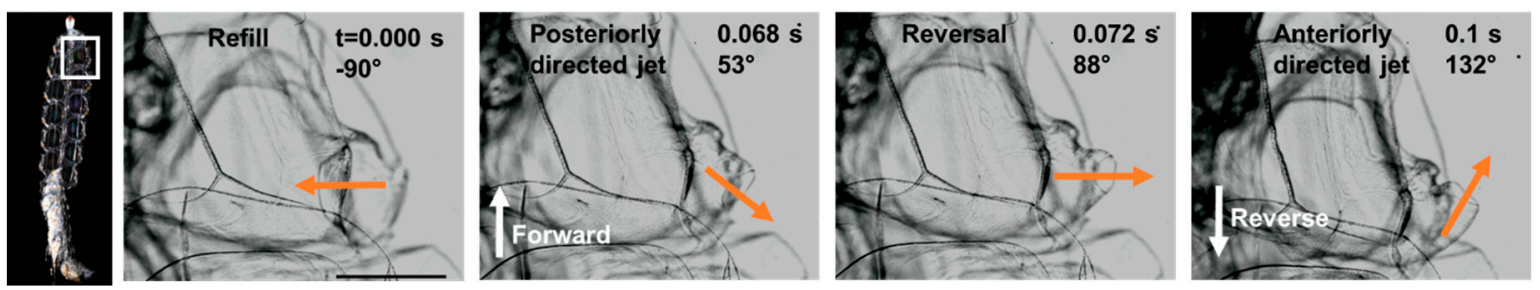

Figure 5. Velum kinematics during transition from forward to reverse swimming with velum angle indicated with orange arrows. Note that a typical jet cycle is either in the forward or reverse direction. Transitions mid-way through a jet are rare but this sequence illustrates the range of velum orientations. White arrows indicate swimming direction. Scale bar in first panel is $1 \mathrm{~mm}$. See Video S4.

\section{Discussion}

Moving in three-dimensional space confers ecological advantages to organisms yet is constrained by morphology and hydrodynamics. N. bijuga is a colonial invertebrate that is highly proficient at a repertoire of swimming maneuvers. Previous investigations highlighted that coordination of nectophores [7] allows for proficient long-distance migrations [11]. Here we detail that maneuvering and reversal are achieved through rapid changes in the velar aperture and fine tuning of the velar angle to tightly control jet production.

Though cnidarian jellyfish are considered to be primitive forms with simple neurocircuitry [12], individual medusae are highly effective swimmers [13]. Cnidarian medusae represent a diversity of forms with some being adept at rapid swimming and others being more effective at slow but efficient swimming $[14,15]$. Individual medusae can also effect turns through manipulation of the bell margin or the velum [12,16]. However, individual medusae cannot achieve backwards swimming due to morphological constraints - they have only one jet orifice and the velum has a limited range of motion for thrust vectoring. The arrangement of multiple units in a colony opens up a much richer array of possible maneuvers. Careful quantification of morphology, body motion and structure-fluid interactions are the first steps towards emulating key maneuvering features in an engineering context.

\subsection{Turning}

Jetting kinematics and fluid mechanics during turning are the same as forward swimming but during turning, pulses are constrained to one or two nectophores at the apex of the colony [7]. A long lever arm and resultant torque is achieved by firing the nectophores at the colony apex. In addition to nectophore position, tight orchestration of velar and nectophore movement achieves impressive maneuverability and agility (Table 1). The length-specific radius of N. bijuga was $0.15 \pm 0.10$ with an absolute minimum value of 0.05. Though other organisms such as squid [17] and box fish [18] have $\mathrm{R} / \mathrm{L}$ values approaching zero, moving forward a tenth or twentieth of a body length during a turn is more than sufficient for maneuvering in most situations. Furthermore, squid and box fish are much more complex than cnidarian swimmers. Aquatic vertebrate swimmers rely on primary and secondary control surfaces along with complex kinematics to move in three-dimensional space [19]. The maximum angular velocity, or agility, of $N$. bijuga was $215 \mathrm{deg} . \mathrm{s}^{-1}$ (Table 1), which is higher than that of many vertebrates with more complex body forms and neurocircuitry (Figure 6). Regardless of phylogeny, organisms must produce thrust along a lever arm in order to turn; N. bijuga achieves this with a simple linear arrangement of nectophores along a linear axis (Figure 1; Video S1). 


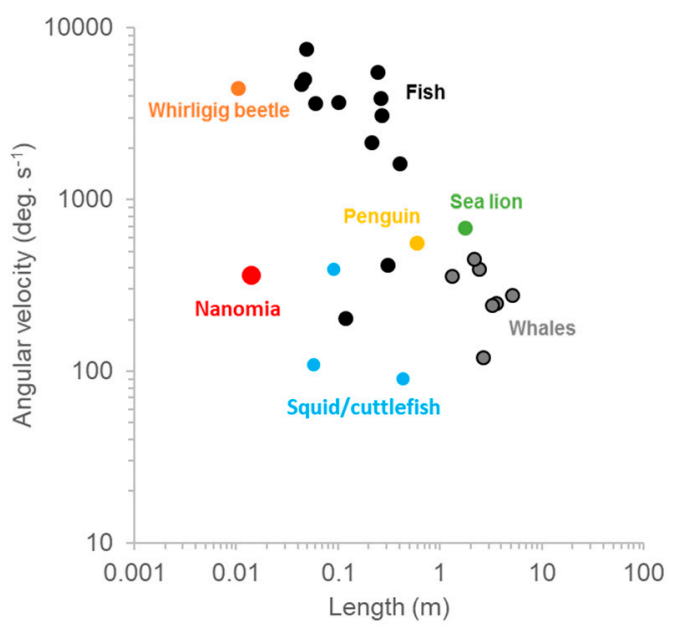

Figure 6. Comparative turning performance in vertebrates and invertebrates. Data are from $[17,20]$ and this study.

\subsection{Reversal}

During reverse swimming the velum changes orientation to redirect the flow anteriorly (Figures 1 and 5; Video S2). Remarkably, swimming during reversal matches forward swimming performance as reported in [8]. Jet speed (two-tailed $t$-test, $t=-1.1 ; \mathrm{df}=12 ; p=0.29$ ), body speed (two-tailed $t$-test, $t=1.6 ; \mathrm{df}=11 ; p=0.14$ ) and jetting time (two-tailed $t$-test, $t=-1.1 ; \mathrm{df}=13 ; p=0.31$ ) are not significantly different between the two swimming modes. Similar performance levels during forward and reverse swimming are likely due to the tight coupling between velum and nectophore kinematics to achieve thrust vectoring. During reverse swimming the velum begins to close in advance of the nectophore (Figure 3) allowing for a narrow, high velocity jet of $\sim 1 \mathrm{~m} \mathrm{~s}^{-1}$ (Table 1) as observed during forward swimming [8]. Having similar levels of performance during forward and reverse motion are rare, even in vertebrates with complex nervous systems. N. bijuga achieves matching forward and reverse swimming speeds with a simple nervous system. One way that forward and reverse swimming differ in N. bijuga is that refill is slower in reverse swimming (two-tailed $t$-test: $t=-2.79, \mathrm{df}=13$, $p=0.015$ ) and as a result the full jet cycle time takes longer during reverse swimming (two-tailed $t$-test: $t=-2.83, \mathrm{df}=13, p=0.015$ ). Reverse swimming is activated as an escape response and was typically limited to a single pulse (see also [6]). During rare instances of multiple reverse pulses, refill time was comparable to jetting time (Figure 3a). Therefore, refill time is not a factor limiting performance during reverse jetting.

The fluid mechanics of refill, however, are a potential constraint on reverse swimming performance. During refill, the jetting ceases, the colony decelerates and there are potential hydrodynamic losses as fluid is pulled into the nectophore. Solitary medusae have been shown to mitigate these losses during refill through a positive pressure region created by the vortex ring generated underneath the bell [13]. Similarly, N. bijuga benefits from a vortex generated inside the nectophore during forward swimming, which generates pressure gradients that enhance forward thrust [8]. During reverse swimming, refill dynamics within the nectophore (Figure 4) match those during forward swimming (Figure 7 in [8]) and would therefore likely counteract reverse thrust. One major difference during refill during forward and reverse swimming is that in forward swimming, fluid is pulled anteriorly to the nectophore and in reverse swimming, fluid is pulled in posteriorly (Figure 4; Video S3); in each case the fluid is pulled in from a direction that would serve to mitigate losses during refill; i.e., decrease thrust opposing the direction of travel.

Fishes and some planktonic invertebrates are competent at reverse swimming. However, most organisms do not achieve reverse swimming speeds that are comparable in magnitude to their forward swimming speed (Figure 7). N. bijuga is notable because the ratio of backward-reverse swimming 
speed is $\sim 1$. This is achieved entirely by simply reorienting the velum anteriorly while the orientation of the rest of the colony and kinematics are unchanged.

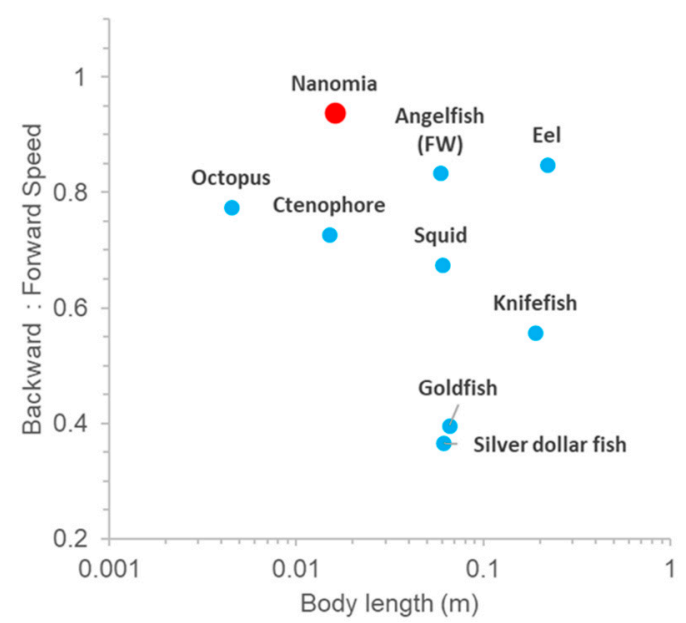

Figure 7. Comparative backward to forward swimming speed ratio in aquatic vertebrates and invertebrates. Squid data show ratio of forward to backward swimming speed since a fins-first, backwards swimming direction is the primary swimming direction. Data are from [21-26] and this study.

\subsection{Neurological Control}

Investigation of kinematics brings up questions about the role of muscle and neuorphysiology in mediating swimming movements. The nectophore is controlled by two sets of muscle fibers-a circular muscle system and a set of radial fibers. The circular muscles control contraction of the nectophore during forward and reverse swimming. The radial fibers of Claus (Claus 1878) contract to pull the velum anteriorly during backward swimming. The fibers of Claus are inactive during forward swimming. Earlier neurophysiological experiments suggested that reverse swimming and forward swimming are controlled by two distinct neurosensory pathways [6]. The forward swimming pathway is excited by stimulation at the base of the colony whereas reverse swimming is excited by stimulation of apical nectophores. The nectophores may experience a sensory transition during development where the younger, apical nectophores evoke reverse swimming and the older nectophores near the colony base evoke forward swimming. Though Mackie [6] suggested that the conduction routes are isolated from one another and that conduction of each is all or none, we made an intriguing observation that the conduction pathway can be interrupted such that the velum reoriented from a posterior directed jet (forward swimming) to an anterior directed jet (reverse swimming) mid-way through a pulse cycle (Figure 5; Video S4). The coordination of the zooids in the colony requires a more sophisticated set of neural pathways but is still attractive from a bio-emulation perspective. The colony follows a set of simple rules that give rise to a diverse array of maneuvers.

\section{Conclusions}

N. bijuga is a highly effective swimmer and a model system for understanding structure-fluid interactions in multi-jet locomotion. It is proficient at long distance swimming but can also perform rapid turns and reversals. Further, effective swimming is achieved with simple cnidarian neurocircuitry. Pelagic colonies are rare, comprising salps and siphonophores, and present unique solutions to aquatic locomotion [27]. The presence of multiple swimming units provides several advantages. Individual units may be small but the colony as a whole can be an order of magnitude larger than any individual unit. Finally, redundancy allows for unaltered whole-colony performance even if individual units are non-functional or lost. The colonial N. bijuga is adept at essentially all aspects of swimming and represents an ideal platform for designing a robust underwater vehicle. N. bijuga is relatively 
small with colony lengths of several $\mathrm{cm}$ and therefore could inspire microrobots; however, physonect siphonophores occur at a range of sizes—up to $10 \mathrm{~m}$ in Stephanomia sp. [28]—suggesting the efficacy of multi-jet propulsion at larger scales.

Supplementary Materials: The following are available online at http://www.mdpi.com/2313-7673/4/3/62/s1, Video S1, N. bijuga turn; Video S2, N. bijuga reverse raw PIV; Video S3, N. bijuga refill PIV; Video S4, N. bijuga thrust vectoring.

Author Contributions: K.R.S., B.J.G., S.P.C. and J.H.C. contributed to collection of the original data and development of analysis methods. K.R.S. performed numerical analysis and wrote the original draft. K.R.S., B.J.G., S.P.C. and J.H.C. contributed to subsequent manuscript revisions.

Funding: This research was funded by the National Science Foundation (NSF) 1829932 and 173764 to K.R.S., NSF 1830015, 1536672, 1511721 to J.H.C., 1455440, 1536688, 1829913 to S.P.C., NSF 1511996 to B.J.G.

Acknowledgments: We are grateful for facilities support from Friday Harbor Laboratories. We thank Sandra Dorning for her help with video analyses. Figure 6 is adapted with permission from the Journal of Experimental Biology ([20]).

Conflicts of Interest: The authors declare no conflict of interest. The funders had no role in the design of the study; in the collection, analyses, or interpretation of data; in the writing of the manuscript, or in the decision to publish the results.

\section{References}

1. Ruiz, L.A.; Whittlesey, R.W.; Dabiri, J.O. Vortex-enhanced propulsion. J. Fluid Mech. 2011, 668, 5-32. [CrossRef]

2. Moslemi, A.A.; Krueger, P.S. Propulsive efficiency of a biomorphic pulsed-jet underwater vehicle. Bioinspir. Biomim. 2010, 5, 1-14. [CrossRef] [PubMed]

3. Serchi, F.G.; Arienti, A.; Laschi, C. Biomimetic vortex propulsion: Toward the new paradigm of soft unmanned underwater vehicles. IEEE ASME Trans. Mechatron. 2012, 18, 484-493. [CrossRef]

4. Mohensi, K. Zero-mass pulsatile jets for unmanned underwater vehicle maneuvering. In Proceedings of the AIAA 3rd “Unmanned Unlimited" Technical Conference, Workshop and Exhibit, Chicago, IL, USA, 20-23 September 2004; Volume 6386.

5. Barham, E.G. Siphonophores and the Deep Scattering Layer. Science 1963, 140, 826-828. [CrossRef]

6. Mackie, G.O. Analysis of locomotion in a siphonophore colony. Proc. R. Soc. Lond. Ser. B Biol. Sci. 1964, 159, 366-391.

7. Costello, J.H.; Colin, S.P.; Gemmell, B.J.; Dabiri, J.O.; Sutherland, K.R. Multi-jet propulsion organized by clonal development in a colonial siphonophore. Nat. Commun. 2015, 6, 8158. [CrossRef] [PubMed]

8. Sutherland, K.R.; Gemmell, B.J.; Colin, S.P.; Costello, J.H. Propulsive design principles in a multi-jet siphonophore. J. Exp. Biol. 2019, 222, jeb198242. [CrossRef]

9. Sutherland, K.R.; Costello, J.H.; Colin, S.P.; Dabiri, J.O. Ambient fluid motions influence swimming and feeding by the ctenophore Mnemiopsis leidyi. J. Plankton Res. 2014, 36, 1310-1322. [CrossRef]

10. Gemmell, B.J.; Jiang, H.; Buskey, E.J. A new approach to micro-scale particle image velocimetry ( $\mu$ PIV) for quantifying flows around free-swimming zooplankton. J. Plankton Res. 2014, 36, 1396-1401. [CrossRef]

11. Robison, B.H.; Reisenbichler, K.R.; Sherlock, R.E.; Silguero, J.M.B.; Chavez, F.P. Seasonal abundance of the siphonophore, Nanomia bijuga, in Monterey Bay. Deep Sea Res. Part II Top. Stud. Oceanogr. 1998, 45, 1741-1751. [CrossRef]

12. Satterlie, R.A. Neuronal control of swimming in jellyfish: A comparative story. Can. J. Zool. 2002, 80, 1654-1669. [CrossRef]

13. Gemmell, B.J.; Costello, J.H.; Colin, S.P.; Stewart, C.J.; Dabiri, J.O.; Tafti, D.; Priya, S. Passive energy recapture in jellyfish contributes to propulsive advantage over other metazoans. Proc. Natl. Acad. Sci. USA 2013, 110, 17904-17909. [CrossRef]

14. Costello, J.H.; Colin, S.P.; Dabiri, J.O. Medusan morphospace: Phylogenetic constraints, biomechanical solutions, and ecological consequences. Invertebr. Biol. 2008, 127, 265-290. [CrossRef]

15. Dabiri, J.O.; Colin, S.P.; Katija, K.; Costello, J.H. A wake-based correlate of swimming performance and foraging behavior in seven co-occurring jellyfish species. J. Exp. Biol. 2010, 213, 1217-1225. [CrossRef]

16. Gemmell, B.J.; Troolin, D.R.; Costello, J.H.; Colin, S.P.; Satterlie, R.A. Control of vortex rings for manoeuvrability. J. R. Soc. Interface 2015, 12. [CrossRef] 
17. Jastrebsky, R.A.; Bartol, I.K.; Krueger, P.S. Turning performance in squid and cuttlefish: Unique dual-mode, muscular hydrostatic systems. J. Exp. Biol. 2016, 219, 1317-1326. [CrossRef]

18. Walker, J.A. Does a rigid body limit maneuverability? J. Exp. Biol. 2000, 203, 3391-3396.

19. Fish, F.E.; Lauder, G.V. Control surfaces of aquatic vertebrates: Active and passive design and function. J. Exp. Biol. 2017, 220, 4351-4363. [CrossRef]

20. Fish, F.E.; Nicastro, A.J. Aquatic turning performance by the whirligig beetle: Constraints on maneuverability by a rigid biological system. J. Exp. Biol. 2003, 206, 1649-1656. [CrossRef]

21. D'Août, K.; Aerts, P. A kinematic comparison of forward and backward swimming in the eel anguilla anguilla. J. Exp. Biol. 1999, 202, 1511-1521.

22. Villanueva, R.; Nozais, C.; Boletzky, S.V. Swimming behaviour and food searching in planktonic Octopus vulgaris Cuvier from hatching to settlement. J. Exp. Mar. Biol. Ecol. 1997, 208, 169-184. [CrossRef]

23. Bartol, I.K.; Krueger, P.S.; Jastrebsky, R.A.; Williams, S.; Thompson, J.T. Volumetric flow imaging reveals the importance of vortex ring formation in squid swimming tail-first and arms-first. J. Exp. Biol. 2016, 219, 392-403. [CrossRef]

24. Webb, P.W.; Fairchild, A.G. Performance and maneuverability of three species of teleostean fishes. Can. J. Zool. 2001, 79, 1866-1877. [CrossRef]

25. Youngerman, E.D.; Flammang, B.E.; Lauder, G.V. Locomotion of free-swimming ghost knifefish: Anal fin kinematics during four behaviors. Zoology 2014, 117, 337-348. [CrossRef]

26. Mackie, G.O.; Mills, C.E.; Singla, C.L. Giant Axons and Escape Swimming in Euplokamis dunlapae (Ctenophora: Cydippida). Biol. Bull. 1992, 182, 248-256. [CrossRef] [PubMed]

27. Mackie, G.O.; Lander, H.J.; Rosen, B.R.; White, J. From aggregates to integrates: Physiological aspects of modularity in colonial animals. Philos. Trans. R. Soc. Lond. B Biol. Sci. 1986, 313, 175-196. [CrossRef]

28. Pugh, P.R.; Baxter, E.J. A review of the physonect siphonophore genera Halistemma (Family Agalmatidae) and Stephanomia (Family Stephanomiidae). Zootaxa 2014, 3897, 1-111. [CrossRef]

(C) 2019 by the authors. Licensee MDPI, Basel, Switzerland. This article is an open access article distributed under the terms and conditions of the Creative Commons Attribution (CC BY) license (http://creativecommons.org/licenses/by/4.0/). 